\title{
Protein S Deficiency and an Adult Case with Moyamoya Syndrome that Presented with Primary Intraventricular Haemorrhage
}

\author{
Betül Çevik ${ }^{1}$, Berat Acu ${ }^{2}$, Dürdane Aksoy ${ }^{1}$, Semiha Kurt ${ }^{1}$ \\ ${ }^{1}$ Department of Neurology, Gaziosmanpasa University Faculty of Medicine, Tokat, Turkey \\ ${ }^{2}$ Department of Radiology, Eskişehir Osmangazi University Faculty of Medicine, Eskişehir, Turkey
}

Background: Moyamoya syndrome associated with protein S deficiency is rarely encountered and is usually reported in paediatric cases with cerebral ischaemia.

Case Report: A 32-year-old woman had symptoms of sudden-onset severe headache, projectile vomiting, impaired consciousness, and slight neck stiffness. The computed tomography scan of her brain showed primary intraventricular haemorrhage, and the subsequent four vessel cerebral angiographies revealed stage 3 to 4 Moyamoya disease according to Suzuki and Takaku's angiographic classification. The coagulation profile showed the presence of protein $\mathrm{S}$ de- ficiency. The patient was treated with external ventricular drainage and conservative management until blood clot resolution. The patient was discharged with normal neurological examination findings after her initial impaired consciousness and orientation defect gradually recovered.

Conclusion: This case report would alert physicians to the possible coexistence of Moyamoya syndrome and protein S deficiency, even in adult cases presenting with primary intraventricular haemorrhage. (Balkan Med J 2014;31:180-3).

Key Words: Intraventricular haemorrhage, Moyamoya syndrome, protein $\mathrm{S}$ deficiency
Moyamoya disease (MMD) is a progressive cerebral vasculopathy characterised by bilateral stenosis or occlusion of the major intracranial cerebral arteries forming the Circle of Willis, which is an extensive network of cerebral collateral vessels $(1,2)$. The pathology is designated as Moyamoya syndrome (MMS) or quasiMoyamoya disease when the same pathological cerebral angiography findings are accompanied by other medical conditions, such as cranial radiation therapy, brain neoplasm, autoimmune diseases, and haematological, prothrombotic, or genetic disorders (1-4).

In the literature, Moyamoya syndrome presenting with ischaemic symptoms accompanied by protein S deficiency (PSD) has been reported in paediatric cases; however, there has been no sufficient data regarding Moyamoya syndrome accompanied by protein S deficiency (PSD) that has been presented with primary intraventricular haemorrhage (PIVH) in adults (5-8). We present here a 32-year-old woman with MMS associated with PSD who developed PIVH.

\section{CASE PRESENTATION}

A 32-year-old right-handed female patient had suddenonset severe headache, projectile vomiting, and impaired consciousness. Computed tomography (CT) scan of her head demonstrated extensive PIVH (Figure 1). External ventricular drainage was applied after a diagnosis of hydrocephalus was established during the follow-up CT scan on the subsequent day. She was referred to our hospital on the $4^{\text {th }}$ day after admission. Her blood pressure was $120 / 75 \mathrm{mmHg}$ and her pulse rate was within 80-90 beats-per-minute. General physical examination did not reveal any abnormalities such as neurocutaneous markers or facial dysmorphism. Glasgow coma score of the patient was 12 . During neurological examination, the consciousness of the patient was somnolent and she could cooperate with simple commands. Place and personal orientation were correct, but time orientation was impaired. The deeptendon reflexes were $3+$ symmetrically. The plantar responses were absent bilaterally. There was slight neck stiffness. Other neurological examination findings were normal. Past medical and family history was unremarkable. Findings on four vessel cerebral angiography (CA) were compatible with Moyamoya disease (Figure 2, 3).

The results of the following investigations were either normal or negative: haemogram (with examination of the peripheral blood smear), sickling test, detailed biochemistry, urine analysis, haemoculture, chest-roentgenogram, electrocardio- 


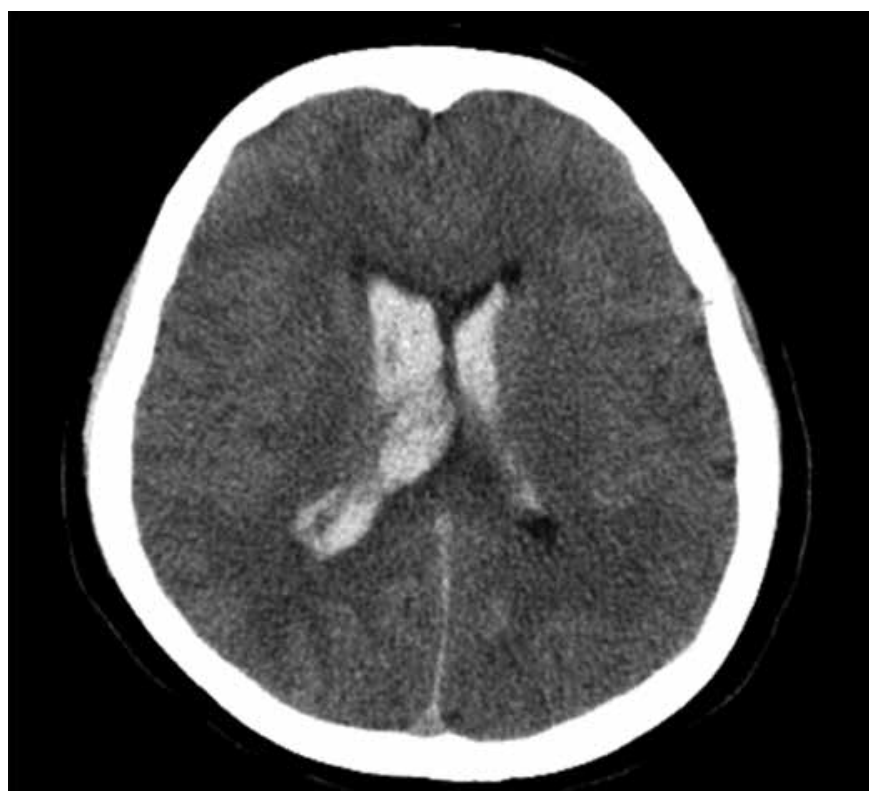

FIG. 1. The unenhanced computed tomography scan of the brain showing extensive intraventricular hemorrhage.

graph, echocardiogram, erythrocyte sedimentation, C-reactive protein, thyroid function tests, homocysteine, ENA panel, c3, $\mathrm{c} 4$, activated protein $\mathrm{C}$ resistance, anti-cardiolipin antibodies (ACA), anti-thrombin III, Factor V Leiden mutation, fibrinogen, lupus anticoagulant (LA), plasminogen, activated partial thromboplastin time, prothrombin time, thrombin time, protein C activity, PR3 ANCA, HIV serology, biochemistry of haemorrhagic cerebrospinal fluid (CSF) taken from the drainage, MTHFR gene mutation, factors V, VIII and XII activity, CSF mycobacterium tuberculosis analysis and culture. Her protein $\mathrm{S}$ level was $11 \%$ (normal value $60 \%$ to $130 \%$ ). Repeat protein $\mathrm{S}$ levels after 3 months were $14 \%$.

The patient's vital signs and electrolyte balance were corrected, and symptomatic medical treatment was applied. Follow-up head CT showed partial resorption of intraventricular haemorrhage, and CSF was clear in drainage. Based on these observations, the drainage tube was removed. Impaired consciousness and orientation defect improved gradually, and the patient was discharged with normal neurological examination findings.

Written informed consent was obtained from the patient who participated in this report.

\section{DISCUSSION}

According to the clinical, radiological, and laboratory findings, the diagnosis of the patient was considered PIVH caused by MMS in association with PSD. PIVH, haemorrhage in the ventricular system without a recognisable parenchymal component, is a rare neurological disorder (9). Moyamoya is defined as a non-atherosclerotic, non-inflammatory, and nonamyloid vasculopathy of unknown aetiology $(1,2)$. In adults, the diagnosis of Moyamoya is defined by 3 angiographic criteria, based on the Japanese Ministry of Health and Welfare guidelines. The first one is stenosis or occlusion at the terminal portion of the internal carotid artery (ICA) and/or at the proximal portion of the anterior and/or the middle cerebral arteries. The second one is abnormal vascular networks in the vicinity of occlusive or stenotic lesions in the arterial phase. The third one is that these findings should be presented bilaterally (4). Vessels of the posterior circulation are rarely involved, as in our case $(2,3)$. CA is still the gold standard for the diagnosis of $\operatorname{MMD}(2,3)$. In our patient, the CA revealed stage 3 to 4 Moyamoya disease according to Suzuki and Takaku's angiographic classification $(1,2)$ (significant ICA narrowing, characteristic puff-of-smoke appearance and development of extra-cranial collaterals), and ruled out vascular malformations. Postpartum cerebral angiopathy, radiation-induced arteritis, and inflammatory angiopathy are important differential diagnoses that may present with cerebral ischaemia and imaging consistent with intracranial vasculopathy mimicking $\operatorname{MMD}(2,3)$.

Although MMD is most common in Japanese and other Eastern Asian individuals, it has been found in all races with varying age distributions and clinical manifestations. The distribution pattern of age of onset has two peaks, with the larger peak in the first decade and the smaller one in the third and fourth decades. Clinically, the presentation of patients with MMD may include transient ischaemic attacks, ischaemic strokes, haemorrhagic strokes, and seizures. Intracranial haemorrhage is common in adults between the ages of 30-50 with a higher occurrence in females and the most frequent site being intraventricular (1-4). In this case report, age, gender, clinical findings and the location of haemorrhage are consistent with previous reports.

Intracranial haemorrhage may occur because of the rupture of saccular aneurysms in the Circle of Willis, resulting in subarachnoid haemorrhage, or because of the rupture of fragile, dilated, and tortuous Moyamoya vessels, yielding intracerebral or IVH (1-3). Rupture of a perforating artery or microaneurysm located in the ventricular subependyma may be another cause of PIVH (2). It was demonstrated by two studies conducted with adult patients with MMD that extension with abnormal branches and excessive dilation of the anterior choroidal and/or posterior communicating arteries (PComAs) are predictors for haemorrhagic lesions, especially IVH (10). There had also been specific dilation on left PComA in angiography in our patient.

Bonduel et al. (5) reported that prothrombotic disorders were detected in 4 out of 10 non-Japanese children with Moyamoya. In their series, inherited PSD was found in 1 patient; LA and ACA were detected in the remaining 3 patients. Pro- 

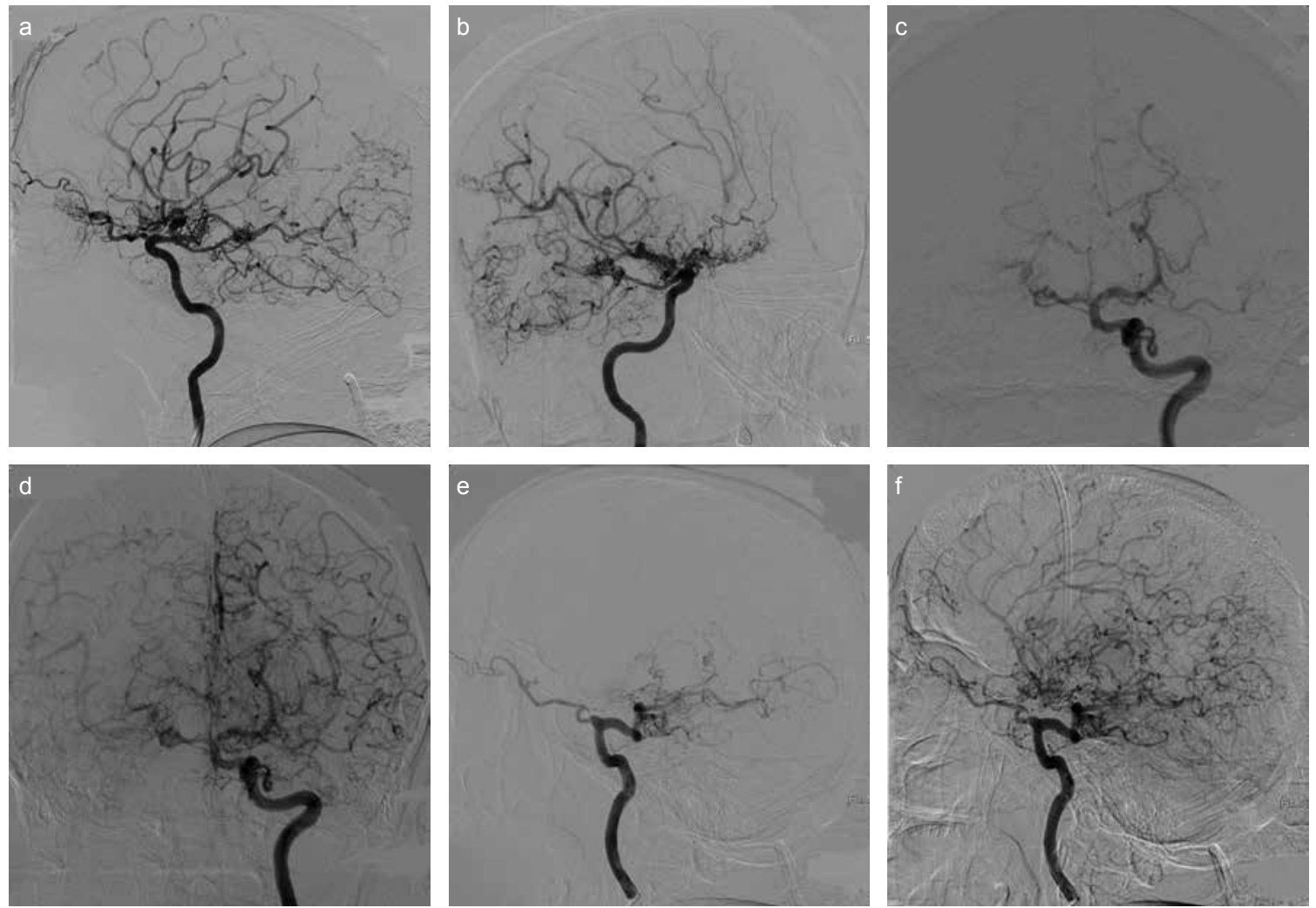

FIG. 2. a-f. Vascular tissue contingent with Moyamoya vessels in lateral projection right ICA injection (a) and 45 degree right anterior oblique position (b). Also noted is the calibre reduction on the right ICA supraclinoid segment and the view belonging to the pial collaterals on the right MCA and PCA distal branches. The left MCA and the left ACA have been totally occluded in the early arterial phase in the left ICA injection in Town projection. It is also noted that the calibre of the supraclinoid segment of the left ICA has become thinner compared to the other ICA segments and shows irregularity (c). The same projection also demonstrates that both ACA and MCA branches show retrograde filling with pial collaterals in the late arterial phase. And moreover, there has been significant dilation of both PComAs (d). In the lateral position, the left MCA and ACA are totally occluded in the early arterial phase, and the left PComA is significantly dilated and fills with the distal basilar and PCA (e). There are bilateral ACA and MCA branches that present pial collateral and retrograde filling on ACA and MCA distal branches in the late arterial phase in the same projection. Additionally, Moyamoya vessels are present around PCA, ACA, and MCA branches (f). (ACA: Anterior cerebral artery, ICA: internal carotid artery, MCA: middle cerebral artery, PCA: posterior cerebral artery, PComA: posterior communicating artery).
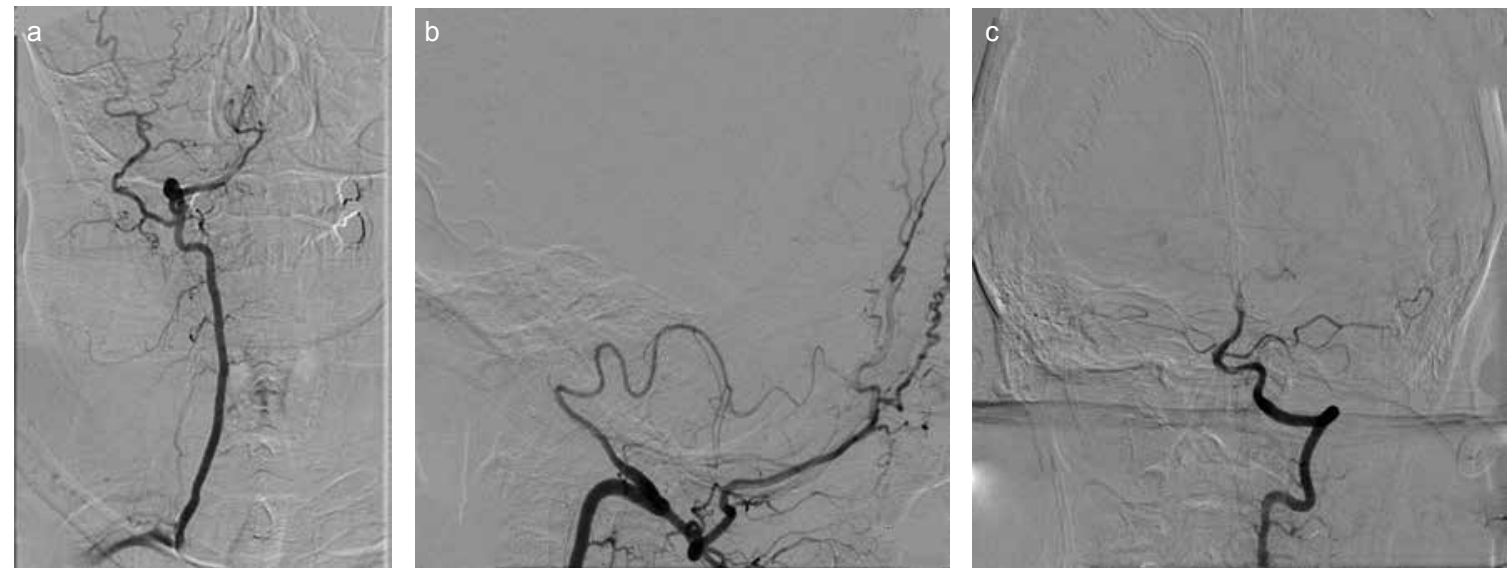

FIG. 3. a-c. In VA in antero-posterior (a) and lateral projection (b), the right VA ends with the PICA with calibre reduction in the intradural segment. Furthermore, the anastomosis between the occipital artery and the extraspinal segment of the right VA is noted. In the left VA Town projection, contour irregularity and calibre reduction in the proximal basilar artery and the left VA intradural segment are noted (c). (PICA: posterior inferior cerebellar artery, VA: vertebral artery). 
tein $\mathrm{S}$ is a vitamin-K-dependent glycoprotein that inhibits the coagulation cascade and functions as a co-factor of activated protein C. PSD is often associated with venous and arterial thromboembolism, and can possibly be related with ischaemic stroke, especially in young patients. It is interesting that our patient had PIVH but not thrombotic disease. Sporadic PSD presents in pregnancy, oral contraceptive use, liver disease, acute inflammation, vitamin $\mathrm{K}$ deficiency, Factor $\mathrm{V}$ Leiden mutation, warfarin treatment, nephrotic syndrome, HIV infection, L-asparaginase chemotherapy or acute thromboembolic events. We ruled out sporadic or transient protein $\mathrm{S}$ deficiency by obtaining repeat protein $\mathrm{S}$ levels after 3 months and with the help of patient history and laboratory findings.

There is no known curative treatment for MMD. In the acute phase of ischaemia and haemorrhagic attack, the treatment is symptomatic (2-4). External ventricular drainage was performed on our patient in whom hydrocephaly had developed, and cerebral perfusion pressure was maintained while avoiding hypovolaemia, dehydration, and hypo/hypertension. Oral anticoagulation is recommended if there is isolated PSD because of the possibility of a recurrent thrombosis. However, prophylactic oral anticoagulation is controversial in MMS patients with PSD due to the risk of intracranial haemorrhage $(5,6)$. Because of PIVH, we postponed anti-coagulation in the acute stage. After the resorption of haemorrhage, the patient was offered anticoagulation; however, this could not be initiated because of inadequate follow-up caused by the patient's non-compliance.

In conclusion, clinicians should consider that PSD can appear not only in Moyamoya patients presenting with ischaemic symptoms, but also in adult Moyamoya patients presenting with haemorrhage.

\section{Ethics Committee Approval: N/A.}

Informed Consent: Written informed consent was obtained from the patient who participated in this case.

Peer-review: Externally peer-reviewed.
Author contributions: Concept - B.C., B.A., D.A.; Design - B.C., B.A., D.A., S.K.; Supervision -B.C., B.A., S.K.; Resource - B.C., B.A.; Materials B.C., B.A.; Data Collection\&/or Processing - B.C., D.A., S.K.; Analysis\&/ or Interpretation - B.C., B.A., D.A.; Literature Search - B.C., S.K.; Writing B.C., S.K.; Critical Reviews - B.C., B.A., D.A.

Conflict of Interest: No conflict of interest was declared by the authors.

Financial Disclosure: The authors declared that this study has received no financial support.

\section{REFERENCES}

1. Burke GM, Burke AM, Sherma AK, Hurley MC, Batjer HH, Bendok BR. Moyamoya disease: a summary. Neurosurg Focus 2009;26:1-10. [CrossRef]

2. Scott RM, Smith ER. Moyamoya disease and moyamoya syndrome. $N$ Engl J Med 2009; 360:1226-37. [CrossRef]

3. Janda PH, Bellew JG, Veerappan V. Moyamoya disease: case report and literature review. J Am Osteopath Assoc 2009;109:547-53.

4. Fukui M. Guidelines for the diagnosis and treatment of spontaneous occlusion of the circle of Willis ('moyamoya' disease). Research Committee on Spontaneous Occlusion of the Circle of Willis (Moyamoya Disease) of the Ministry of Health and Welfare, Japan. Clin Neurol Neurosurg 1997;99:238-40. [CrossRef]

5. Bonduel M, Hepner M, Sciuccati G, Torres AF, Tenembaum S. Prothrombotic disorders in children with moyamoya syndrome. Stroke 2001;32:1786-92. [CrossRef]

6. Tsuda H, Hattori S, Tanabe S, Nishioka S, Matsushima T, Ikezaki K, et al. Thrombophilia found in patients with moyamoya disease. Clin Neurol Neurosurg 1997;99:229-33. [CrossRef]

7. Charuvanij A, Laothamatas J, Torcharus K, Sirivimonmas S. Moyamoya disease and protein S deficiency: a case report. Pediatr Neurol 1997;17:171-3. [CrossRef]

8. Akgün D, Yılmaz S, Senbil N, Aslan B, Gürer YY. Moyamoya syndrome with protein S deficiency. Eur J Paediatr Neurol 2000;4:185-8. [CrossRef]

9. Flint AC, Roebken A, Singh V. Primary intraventricular hemorrhage: yield of diagnostic angiography and clinical outcome. Neurocrit Care 2008;8:330-6. [CrossRef]

10. Liu W, Zhu S, Wang X, Yue X, Zhou Z, Wang H, et al. Evaluation of angiographic changes of the anterior choroidal and posterior communicating arteries for predicting cerebrovascular lesions in adult moyamoya disease. J Clin Neurosci 2011;18:374-378. [CrossRef] 